of America, 1970). The discovery of man-made earthquakes inevitably raised the question of how they were produced; and this led to a basic understanding of the part played in earthquake mechanisms by fluids. Meanwhile, several workers, beginning with Mogi (Bull. Earthquake Res. Inst., $40,125 ; 1962)$ and continuing with Brace (Tectonophysics, 6, 75; 1968) and Byerlee (J. geophys. Res., 72, 3639; 1967) and others, had been investigating the behaviour of rocks under stress in laboratory conditions, and had come to appreciate the importance of fracturing in rocks in earthquake-prone regions.

All three developments finally came together during 1972-73 in physical models proposed by Nur (Bull Seismol. Soc. Am., 62, 1217; 1972), Scholz et al. (Science, 181, 803; 1973) and Whitcomb et al.-models which differ only in detail. In that of Whitcomb et al., for example, the pores and cracks in the initially unstrained rock are saturated with fluid. As the stress builds up prior to an earthquake the rock increases in volume (dilates), leading to a decrease in pore fluid pressure and ultimately to a situation in which the pore and crack volume exceeds the fluid volume. In this undersaturated state the rock voids partially contain vapour, which reduces the overall bulk modulus, $V_{P}$ and hence $V_{\mathrm{p}} / V_{\mathrm{s}}$. The initial effect of the dilatancy is thus to delay the onset of the earthquake (dilatancy strengthening); but fluid from outside the dilatant volume gradually flows in, returning the volume to its saturated state, increasing $V_{\mathrm{P}} / V_{\mathrm{S}}$ and decreasing the fracture strength until the earthquake occurs.

With a promising prediction technique to be developed
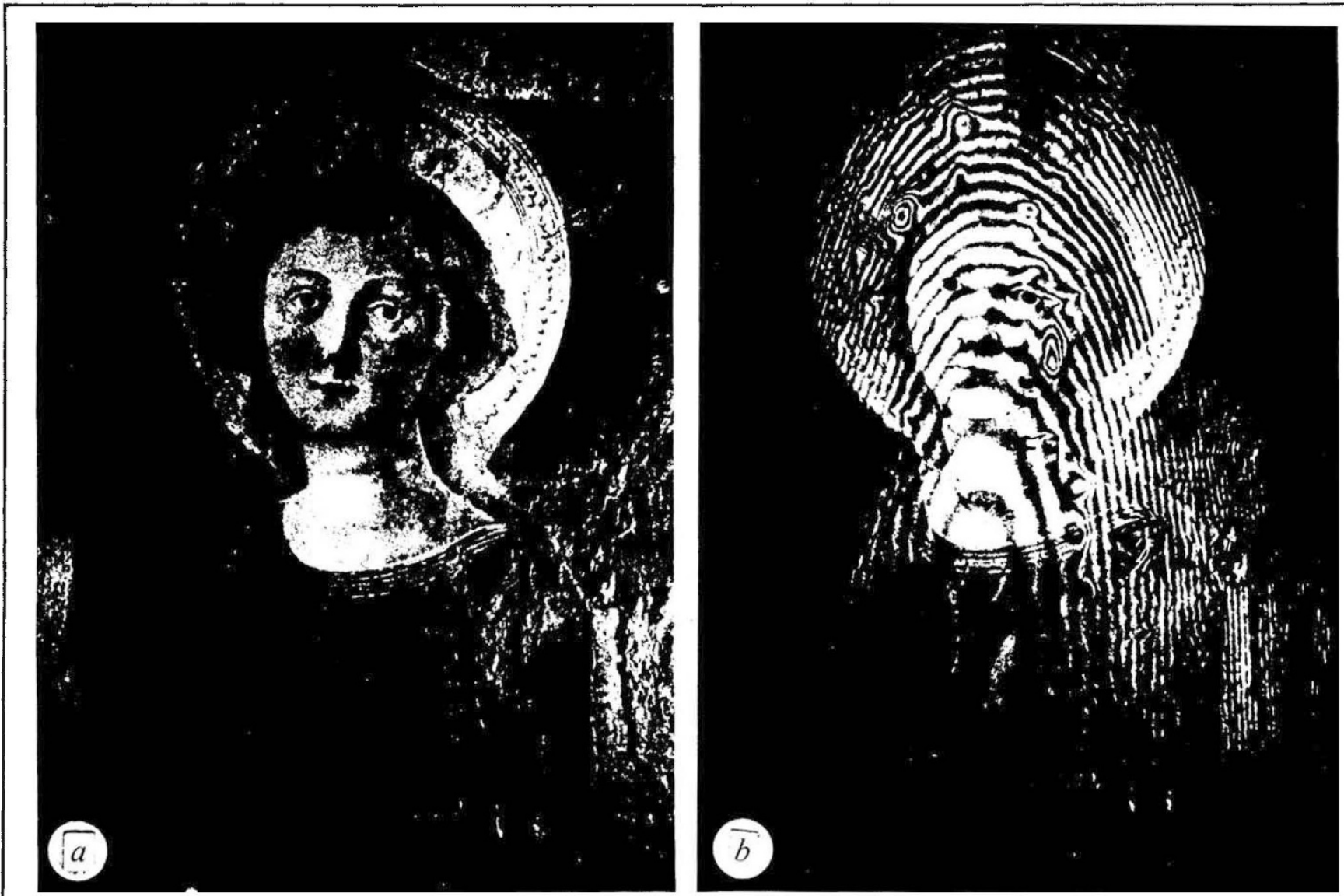

Fig. $1 a$, Santa Caterina by Pier Francesco Fiorentina; $b$, damaged areas indicated by holographic interferometry.

THE detection of incipient damage and deterioration in oil paintings is important for their conservation and restoration. A technique developed by Italian scientists will make this easier in future. S. Amadesi et al. (Applied Optics, 13, 2009; 1974) used holographic interferometry to study paintings on wooden panels. Such pictures have several layers of primer underneath the paint, and these are liable to peel away from the wood. In the early stages the detachment is easier to repair but harder to detect.

Amadesi and his colleagues realised that if a painting were warmed, detached regions would disperse heat at a lower rate than the surrounding areas, so their temperature rise and thermal expansion would be greater.

\section{Painting holograms}

\section{from John Walker}

These changes could then be observed by taking two holograms at fiveminute intervals, using a laser. The principle is like superimposing two almost identical photographs: any differences are immediately apparent. The advantage of holograms is that much smaller differences are observable.

In a test run on a laboratory specimen-primed poplar wood--plastic slivers under the primer were easily located. The crucial test was made on the fifteenth century Italian panel painting shown in Fig. 1a. The change in room temperature during the five minutes between the two holograms was sufficient to indicate some detachments, but localisation was poor. So the painting was warmed to $40^{\circ} \mathrm{C}$ (not hot enough to cause damage). Many more detachments were then observed, and they were better defined. This is shown in Fig. $1 b$. The 'contour lines' superimposed on the image are interference fringes, and the kinks indicate detached regions. In undamaged paintings these distortions of the fringe pattern do not occur.

The method can also be applied to frescoes and paintings on canvas. It will be increasingly useful, not only to locate detachments already present, but to monitor undamaged paintings to make sure they stay that way. 